\title{
Artificial Intelligence Mechanisms on Interactive Modified Simplex Method with Desirability Function for Optimising Surface Lapping Process
}

\author{
Pongchanun Luangpaiboon and Sitthikorn Duangkaew \\ Industrial Statistics and Operational Research Unit (ISO-RU), Department of Industrial Engineering, \\ Faculty of Engineering, Thammasat University, Pathum Thani 12120, Thailand
}

Correspondence should be addressed to Pongchanun Luangpaiboon; lpongch@engr.tu.ac.th

Received 6 June 2014; Revised 20 August 2014; Accepted 16 September 2014; Published 2 October 2014

Academic Editor: Rui Mu

Copyright (C) 2014 P. Luangpaiboon and S. Duangkaew. This is an open access article distributed under the Creative Commons Attribution License, which permits unrestricted use, distribution, and reproduction in any medium, provided the original work is properly cited.

A study has been made to optimise the influential parameters of surface lapping process. Lapping time, lapping speed, downward pressure, and charging pressure were chosen from the preliminary studies as parameters to determine process performances in terms of material removal, lap width, and clamp force. The desirability functions of the-nominal-the-best were used to compromise multiple responses into the overall desirability function level or $D$ response. The conventional modified simplex or Nelder-Mead simplex method and the interactive desirability function are performed to optimise online the parameter levels in order to maximise the $D$ response. In order to determine the lapping process parameters effectively, this research then applies two powerful artificial intelligence optimisation mechanisms from harmony search and firefly algorithms. The recommended condition of (lapping time, lapping speed, downward pressure, and charging pressure) at $(33,35,6.0$, and 5.0$)$ has been verified by performing confirmation experiments. It showed that the $D$ response level increased to 0.96 . When compared with the current operating condition, there is a decrease of the material removal and lap width with the improved process performance indices of 2.01 and 1.14, respectively. Similarly, there is an increase of the clamp force with the improved process performance index of 1.58 .

\section{Introduction}

The hard disk drive (HDD) precision components mainly consist of magnetic, mechanical, electromechanical, and electronic components. A built-in hard drive of a disk clamp on the spindle motor hub assembly is one of important mechanical components in the HDD. The surface quality of the disk clamp in contact with the disk needs to concentrate via a surface lapping process. It is the precision finishing process with the different mechanical arrangement where a material is precisely removed from a work piece or a specimen. It aims to produce a desired dimensional accuracy and flatness, very fine surface finish, or shape with minimal level of subsurface damage [1]. Lapping is an operation of slow material removal [2]. It aims to decrease the original surface roughness with preset levels of removed material and modified shape. A process of lapping has been applied to a wide range of materials and applications, ranging between metals, glass, optics, semiconductors, and ceramics [3]. In lapping operations the machining methods can be practically categorised into cylindrical lapping between laps, lapping with bonded abrasives and single and double side flat lapping types.

With the rapid development of the HDD industries there is consequently a need for enhancing all related performance measures of lapping operations. It is more challenging to achieve better surface quality of disk clamps with high manufacturing efficiency. In order to successfully control the process, it is important to analyse all the influential parameters and determine their proper levels in manufacturing processes. Many studies have been performed on various methods of automatic optimisation based on surface lapping process parameters. Response surface methodology (RSM) was widely used to determine and predict the optimal 


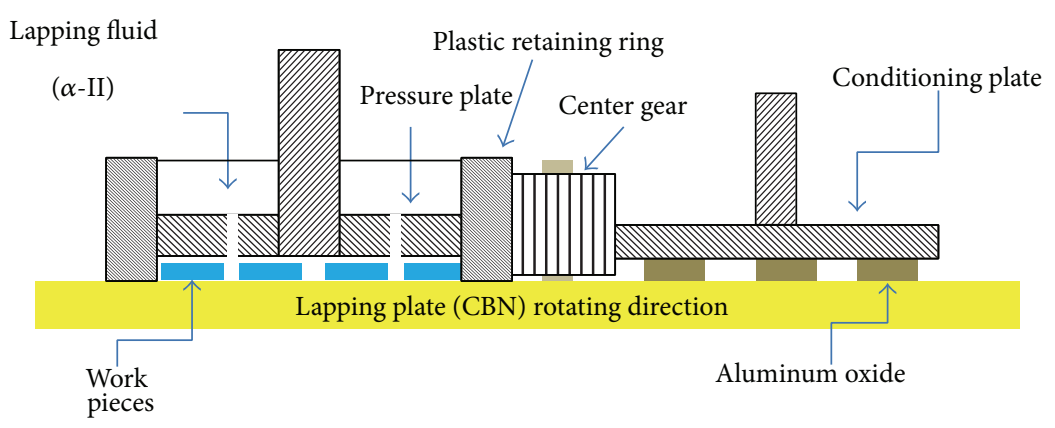

FIGURE 1: Schematic of the section view of surface lapping process.

parameter levels for the maximal improvement of the surface roughness for the cylindrical lapping of fine ceramics [4]. This cylindrical lapping was characterised and optimised because it was time consuming and very complicated. Other powerful tools for designing high quality systems were based on Taguchi designs and analyses $[5,6]$. When optimising performance, quality and cost Taguchi's strategy was simple, efficient and systematic. The smaller-the-better quality characteristic was used to determine minimal surface roughness when lapping ceramic blocks. The influential lapping parameter was the abrasive size [7]. The experimental design and analysis including the regression were conducted on a study on surface roughness and material removal rate of D2 steel lapping with a polymer-coated plate. From an investigation the effects and optimal levels of process parameters were lapping time, lapping rotation speed, applied load, and abrasive particle size [8].

\section{Surface Lapping Process (SLP)}

One of the best known and most widely used machining systems for manufacturing the desired dimension of engineering parts is a single side lapping process. There are some advantages for this type of the lapping process. During machining many work pieces can be simultaneously carried out. The system for holding work is not complicated. It provides consistent cut rates and close accuracies. All machines used in the single side lapping process have a rotating annular-sharped lap plate. Work pieces or disk clamps are positioned on the flat rotating wheel as shown in Figure 1. Lapping mainly includes lapping plate and fluid including the conditioning ring [3]. Operational steps of the lapping process are followed. Firstly, parts or disk clamps are loaded into carriers. The plastic retaining ring and neoprene are then placed to control the parts at the right controlled area. A flat aluminum plate is placed on top of neoprene to protect the work pieces from the pressure plate via a suction cup. Retaining ring slides onto a lapping plate to actuate the pressure plate down. Parts are lapped and coolants are used as lubricants. The process simultaneously applies the downward pressure on a disk, charging pressure of $\mathrm{Al}_{2} \mathrm{O}_{3}$, speed up the lapping plate and lapping time. The parts are finally unloaded to clean and dry.

Some important functions and features of SLP components are briefly explained as follows. Lapping plates are made of cubic boron nitride or CBN with the size of $20 \mu \mathrm{m}$ and hexagonal tiles of $92 \%$ minimal coverage. The types of the lapping fluid and the conditioning plate are the cutting fluid Alpha-2 and $\mathrm{Al}_{2} \mathrm{O}_{3}$ with the grit size number 220, respectively. A human-made synthetic abrasive of $\mathrm{CBN}$ is commonly known as borazon TM CBN. It performs well to ferrous metal without carbonisation when interacting with Fe (iron) during a lapping operation of 52100 bearing steel, cast iron, die steel, tool steel, super alloys, and some cases of ceramic materials [3]. Lapping fluid provides an important carrier characteristic in forms of an oil or aqueous medium in various viscosities when abrasive grains are transported to the lapping operation zone. An aim is to achieve a continuous distribution across the lapping plate. The liquid carrier is applied to lubricate two surfaces to achieve the friction reduction between the abrasive and the work piece [3]. It helps to distribute the abrasive product across the lapping plate and finally to remove the abraded debris from the lapping operation zone. The structure of a conditioning plate of a fused crystalline abrasive of Mohs 9 under silicon carbide has very hard crystal to fracture [3]. On lapping operations it is best suited for applying the pressure to break down the crystals.

In this research, the surface lapping process is used to produce dimensionally accurate specimens or the disk clamps to high tolerances. The lapping plate will rotate at the low levels of speed, approximately less than $80 \mathrm{rpm}$, and the middle range of abrasive particles of 5-20 $\mu \mathrm{m}$. With the preferable levels of reliability and produced work pieces lifetime, the material removal of work pieces including a lap width and a desired clamp force are SLP responses to compromise process and customer specifications within their lower and upper levels. Under the current operating condition, the mean, standard deviation and an overall process performance index (Ppk) of all responses of material removal, surface lap width, and clamp force have unsatisfactory levels when compared to the target (Table 1).

In order to satisfy the customers and get the higher levels of benefit, the investigation and improvement for this process is carried out. The sequential experiments of completely randomised design and the two-level factorial design were applied in a preliminary study to determine the influential parameters via all actual responses. Lapping processes have a large number of parameters that can be varied in order to obtain the desired process responses. The lapping process 
TABLE 1: Quality characteristics at the current operating condition.

\begin{tabular}{|c|c|c|c|c|c|c|}
\hline \multirow{2}{*}{ Response } & \multicolumn{3}{|c|}{ Current statistics } & \multicolumn{3}{|c|}{ Specification } \\
\hline & Mean & Standard deviation & Ppk & Lower & Target & Upper \\
\hline Material removal & 1.383 & 0.134 & 0.91 & 0.25 & 1 & 1.75 \\
\hline Lap width & 0.767 & 0.028 & 0.95 & 0.45 & 0.65 & 0.85 \\
\hline Clamp force & 26.54 & 0.585 & 0.88 & 25 & 28 & 31 \\
\hline
\end{tabular}

is influenced by lapping pressure, lapping speed, material of the lapping plate, lapping time, grain size of the abrasive, type of lapping fluid, flow rate, and the number of work pieces. It is almost impossible to vary all the parameters that influence the lapping process; one solution is to limit the number of variables. The desirability function approach in the case of the-nominal-the-best was used to compromise the multiple responses of material removal, lap width, and clamp force into single response in forms of the composite desirability function level or $D$. The modified simplex method was implemented online to drive the process achieving the optimal condition. When there was no improvement from the modified simplex method, an interactive desirability function model was also applied to obtain the satisfactory compromise of the conflict responses instead of the original massive contraction. Artificial intelligence mechanisms of the harmony search and firefly algorithms were generated in each design point of the simplex to search for the better representative. Its aim was to converge to the optimum quickly based on the maximal $D$ levels.

\section{Interactive Modified Simplex Method (IMSM) with Desirability Function and Artificial Intelligence Mechanisms}

Design and analysis of experiments (DOE) is determined as a sequential, structured, and efficient framework for planning experiments and then describing the relationship between influential parameters affecting a process or product and its output or response(s) in any experimental context. Practically, one or several parameters at two or more levels will be varied by experimenters and the aim is to observe the effect of the changes on one or multiple responses. A frequent phenomenon of this interaction between parameters is taken into account. There is a theoretical argument that the minimal number of trials for finding this relationship is just one more than the number of parameters, that is, a simplex design. On both single and multiple responses, the sequential procedures based on the simplex designs involve changing all parameters from one experiment to the next. The preferable level for one of them can depend on other parameter levels. However, some trials deteriorate the process yield or the composite desirability function level for the multiple response surface optimisation. Various methods have been proposed to solve a large number of optimisation problems and some are considered as NP-complete problems or noisy problems. Conventional optimisation methods are useful tools to obtain the global optimum though some methods are unable to solve within satisfactory execute time. These conditions and difficulties have forced to develop alternative methods of artificial intelligence for solving such problems via various rules of randomness or natural phenomena such as natural biological evolutionary process or the social behavior of species. Artificial intelligence methods came to the fore and show great efficiency when solving problems. Artificial intelligence methods are commonly categorised into two types of a local and population based methods when searching for the global optimal solution $[9,10]$. These mechanisms including an interactive desirability function model have been proposed to prevent the mature convergence of the process parameters.

3.1. Desirability Function Approach (DFA). In robust designed experiments there is a use of measurements on a set of design points with various responses [11]. Instead of optimising each response separately, settings for the influential parameters sought to satisfy all of the responses at once. Multiple response surface optimisation is then used to determine optimal levels of process or product parameters with low variation of responses [12]. There are some transformation scenarios to deal with multiple responses. One among the most frequently used multiple response optimisation strategies in practice is a desirability function approach or DFA which was originally developed by Harrington and modified to be more flexible by Derringer and Suich. It is a scale invariant index or a single compromise function from multiple responses to enable to compare quality characteristics with various units. The DFA basic idea involves transformation of a multiple response problem into a single response problem, by using proper mathematical transformations, to simultaneously optimise those multiple responses. The DFA introduces, for each estimated response determined by the $X$ vector of process parameters, a desirability function of $d_{i}\left(\hat{y}_{i}\right)$ with feasible levels between zero and one where the desirability level of one represents the closeness of a response to its most desirable level. If the $i$ th response falls within the unacceptable levels the desirability level turns to zero. When a response lies within the tolerance levels but not the ideal levels, the desirability level lies between 0 and 1. There are two cases of one-sided and two-sided desirability transformations when each estimated response of $\widehat{y}_{i}$ is transformed to $d_{i}\left(\hat{y}_{i}\right)$.

According to the $m$ responses' characteristics there are three forms of the desirability function which consist of the maximisation or the larger-the-best or the minimisation or the smaller-the-best and the optimisation around a target or the-nominal-the-best. For the larger-the-best the response level is expected to the larger the best. When the response 
TABLE 2: Transformation parameters of the DFA.

\begin{tabular}{ll}
\hline Parameter & Definition \\
\hline$\widehat{y}_{i}$ & ith estimated response model \\
$Y_{i}^{\text {MIN }}$ & Minimal acceptable values of the $i$ th response \\
$Y_{i}^{\text {MAX }}$ & Maximal acceptable values of the $i$ th response \\
$T_{i}^{\text {MIN }}$ & Lower targets of the $i$ th response \\
$T_{i}^{\text {MAX }}$ & Upper targets of the $i$ th response \\
\hline
\end{tabular}

level excesses a preset criteria level or its requirement the desirability function level is equal to 1 . The desirability function of the larger-the-best can be written as follows:

$$
d_{i}\left(\widehat{y}_{i}\right)= \begin{cases}0, & \text { if } \widehat{y}_{i} \leq Y_{i}^{\mathrm{MIN}}, \\ {\left[\frac{\widehat{y}_{i}-Y_{i}^{\mathrm{MIN}}}{Y_{i}^{\mathrm{MAX}}-Y_{i}^{\mathrm{MIN}}}\right]^{\mathrm{PM}_{i},},} & \text { if } Y_{i}^{\mathrm{MIN}} \leq \widehat{y}_{i} \leq Y_{i}^{\mathrm{MAX}} \\ 1, & \text { if } \widehat{y}_{i} \geq Y_{i}^{\mathrm{MAX}} .\end{cases}
$$

For the smaller-the-best the response level is expected to be the smaller-the-best. When the response level is less than a preset criteria level, the desirability function level is equal to 1 . In contrast if the response level excesses a preset criteria level, the desirability function level equals 0 , where the smaller-the-best is equivalent to the maximisation of $-\widehat{y}_{i}$. For the-nominal-the-best the response level is required to achieve a particular target. When the response level equals the target, the desirability function level equals 1 . When there is a departure of the response level over a particular target range, the desirability function level equals 0 . The desirability function of the-nominal-the-best can be written as follows and the associated effects of the weights are also included in Table 2:

$$
\begin{aligned}
& d_{i}\left(\widehat{y}_{i}\right)
\end{aligned}
$$

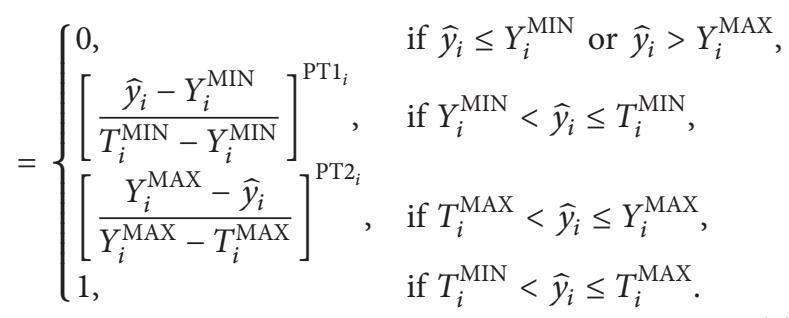

According to the requirement of the user the shape of $d_{i}\left(\hat{y}_{i}\right)$ is determined via the power coefficients of $\mathrm{PM}_{i}, \mathrm{PT}_{i}$, and $\mathrm{PT} 2{ }_{i}$. With the linear shape $\mathrm{PT} 1_{i}$ or $\mathrm{PT} 2_{i}$ equals one. With a convex $\mathrm{PT} 1_{i}$ or $\mathrm{PT} 2_{i}$ is larger than 1 and with a concave $\mathrm{PT} 1_{i}$ or $\mathrm{PT} 2_{i}$ is less than 1 . For the-nominal-the-best, if $T_{i}^{\mathrm{MIN}}$ equals $T_{i}^{\mathrm{MAX}}$ on (2), a triangular desirability function will be applied instead of the trapezoidal function [13]. When each of the responses of interest is defined as the desirability function levels, an overall assessment of all the desirability functions in forms of the geometric mean of the desirability function levels or $D$ is followed:

$$
D=\left(\prod_{i}^{m} d_{i}\left(\widehat{y}_{i}\right)\right)^{1 / m}
$$

$D$ or the composite desirability function level provides a mean level less than or equal to the lowest individual optimisation desirability level. $D$ will increase as the balance of the properties is more favourable regardless of the values taken on by other responses [14]. The objective is to maximise $D$. When the $D$ level is different from zero it is implied that all responses are simultaneously in a desirable level. Consequently, for the $D$ level close to 1 , it is implied that the combination of the different criteria is globally optima or the response levels are simultaneously near the target values.

\subsection{Interactive Desirability Function Approach (IDFA). A} decision maker (DM) determines this combined dimensionless desirability function for constructing the sequential optimisation procedures. With this potential effectiveness for compromising all responses various works attempt to solve multiple response surface optimisation problems in an interactive manner via a DM's preference parameters such as the shape, bound, and target of a desirability function in both single and integrated frameworks. In the progressive preference or interactive method the DM can progressively articulate the preference information while solving the problem in forms of interactive desirability function approach (IDFA). For sequential procedures of the IDFA it starts from an initialisation phase. The calculation phase is then constituted and followed by the decision-making phase via an optimisation model. During the decision-making phase, the numerical results of the calculation phase are evaluated and articulated the preference information by the DM [13]. The DM can adjust the shape, bound, or target of a desirability function on either tightening or relaxation to repeat the calculation procedure until there is the DM satisfaction via the intersection of desirability functions corresponding to actual responses. The modes of tightening and relaxation are applied when the DM makes the unsatisfactory requirements more stringent and the satisfactory requirements less stringent, respectively. In the IDFA the preference parameters such as shape, bound, and target need to be initialised to construct the desirability function in the first iteration. The initial shape is practically set to be linear whereas the initial bound and target depend on the DM's subject judgments or the feasible ranges of the responses. The iteration starts at zero and the response indices of the tightening or relaxation set is set at null. However, for simplicity it is assumed that merely one response can be tightened or relaxed at a time. With an iteration increase, the desirability functions are then constructed and the optimisation model is solved and subject to process parameters $(X)$ within their lower (LB) and upper (UB) bounds. An objective is to maximise the overall desirability among various aggregation schemes to be employed. For three actual responses, an interactive 
desirability function model (IDFM) is then formally defined as

$$
\begin{array}{ll}
\text { Maximise } & D \\
\text { Subject to } & d_{1}\left(\widehat{y}_{1}\right) \geq D, \\
& d_{2}\left(\widehat{y}_{2}\right) \geq D, \\
& d_{3}\left(\widehat{y}_{3}\right) \geq D, \\
& D \geq 0 \\
& \mathrm{LB}<X<\mathrm{UB} .
\end{array}
$$

If the current solution is evaluated to reach the optimal compromise solution the IDFA successfully ends. Otherwise, the algorithm moves to adjust the preference parameter in order to improve the unsatisfactory responses. On the DM selection, one of the unsatisfactory responses can be chosen for tightening or one of the satisfactory responses for relaxation. Both tightening and relaxation modes of the selected response can be implemented in the IDFM by adjusting the preference parameters. For the-nominal-thebest the tightening is implemented by making the shape more convex or less concave, by increasing the lower target and/or decreasing the upper target or by increasing the lower bound and/or decreasing the upper bound. The IDFA restarts until the DM is unwilling to tighten or relax any response or no further tradeoff can be made.

3.3. Harmony Search Algorithm (HSA). The HSA, firstly proposed by Lee and Geem, in 2001, is a population based or socially-based inspiration algorithm. It is derived from the behaviour of musicians when they all improvise their various musical instruments to achieve a perfect state of harmony [15]. In the musical improvisation, the aesthetic quality is conceptually determined via the pitch played by each instrument [16]. Moreover, after practice of musicians the harmony quality can be enhanced for all types of musical instruments. Each musician stores all pitches from a good experience of harmony and for the next time it is possible to have a better level. In all the musicians' memories each musician brings any preferable pitch in the possible range to improvise each other to form a new musically harmony vector. If this leads to the better one when compared to existing harmonies in their memories a new harmony is replaced in their memories. With a controlled number of pitches the worst one is also excluded from their memories. There are three possible rules to improvise for each musician [10]. They are rules of playing any pitch from a musician's memory, playing an adjacent pitch of one pitch from a musician's memory, or randomly playing any pitch from the feasible sound range. This process is repeated until there is a fantastic harmony or the termination criterion is met.

Similarly, the improvisation is analogous to the global search scheme in the HSA. For the optimisation the global optimal solution is determined via the levels of a set of parameters. Each musician can be replaced with each parameter and the harmony is analogous to the response of the problem. With an experience to seek for the optimum, any good solution is stored in each parameter's memory. For the next iteration there is an increase of a possibility to make a good solution. In order to choose one parameter level to improve the $D$ response the HSA follows three rules as above. They consist of rules for choosing any one level from the harmony memory (HM) within the specific harmony memory size (HMS), choosing an adjacent level of any one in the HM which is called the pitch adjustment with an arbitrary distance bandwidth (BW), or randomly choosing any level from the possible range which is defined as randomisation. The optimisation via improvisation is associated with a harmony memory considering rate $\left(P_{\mathrm{HMCR}}\right)$ and a pitch adjusting rate $\left(P_{\mathrm{PAR}}\right)$ parameters. The movement of the $i$ th parameter is adjusted to improvise more harmony by the equation of $x_{i+1}=x_{i} \pm$ (rand)BW(ITE), where is BW(ITE) $=$ $\mathrm{BW}_{\text {MAX }} \operatorname{EXP}\left(I\left(\ln \left(\mathrm{BW}_{\mathrm{MAX}} / \mathrm{BW}_{\mathrm{MIN}}\right) / \mathrm{ITE}\right)\right)$. In the literature for the HSA parameter levels, the $\mathrm{BW}_{\mathrm{Max}}, \mathrm{BW}_{\mathrm{Min}}$, HMS, $P_{\mathrm{HMCR}}$ and $P_{\mathrm{PAR}}$ are recommended to be within the range of $1,[0.001,0.1],[20,50],[0.7,0.95]$ and $[0.3,0.7]$, respectively.

3.4. Firefly Algorithm (FA). Fireflies naturally generate light from a chemical process in light-emitting organs. The light in adult fireflies is evolved to communicate for a sexual selection and to search for potential preys via varied flashing patterns, the flashing rate, and the amount of time of the signal system. At a specific distance from the light source its intensity follows the inverse square law or when there is an increase of the distance the light intensity decreases. Moreover, the light becomes weaker from the air effect when there is a distance increase. These combined factors make most fireflies visible to communicate with each other within a limited distance. Similarly, the flashing light of the successful communication is analogous to the global search scheme in the FA. For the optimisation the global optimal solution is determined via the levels of a set of parameters. The flashing behaviours of fireflies in nature consequently develop the firefly artificial intelligence algorithm (FA) for the global search scheme, firstly developed by Yang $[17,18]$. There are some important characteristics in describing the FA as follows. Other fireflies with weaker flashes are attracted by a strong flashing firefly regardless of their sex. That attractiveness is proportional to the brightness and is reversely proportional to the distances. As a result for any two flashing fireflies, the less bright one will move towards the brighter one. If there is no brightness difference, the firefly will move randomly. In the FA, the brightness of a firefly is proportionally determined by the $D$ response.

In the FA there are two important issues which are the formulations of brightness and attractiveness. For simplicity, the brightness of a firefly at a particular location or $I(x)$ is analogous to the response of a solution at a particular parameter level or $f(x)$. The attractiveness or $\beta$ by the brightness or the higher $D$ response level of a firefly or a specific solution is relative and judged by the other fireflies or other solutions. So the attractiveness function or $\beta\left(r_{i j}\right)$, whose value decreases with increasing distance between two fireflies, will vary with the distance between $i$ th and $j$ th fireflies $\left(r_{i j}\right)$ by any monotonically decreasing functions 
such as $\beta(r)=\beta_{0} e^{-\gamma r_{i j}^{2}}$ or $\beta_{0} / 1+\gamma r_{i j}^{2}$. The parameters of $\beta_{0}$ and $\gamma$ are the attractiveness at $r_{i j}=0$ and a light absorption coefficient or the variation of the attractiveness in the environment, respectively. The $\gamma$ value is crucially important in determining the convergence speed and the FA behaviour. Over $d$ dimensions, the distance of $r_{i j}$ between $i$ th and $j$ th fireflies at $x_{i}$ and $x_{j}$ can be the Cartesian distance function of $r_{i j}=\left\|x_{i}-x_{j}\right\|=\sqrt{\sum_{k=1}^{d}\left(x_{i k}-x_{j k}\right)^{2}}$, where $x_{i k}$ is the $k$ th component of the spatial coordinate of $x_{i}$ for the $i$ th firefly. The movement of the $i$ th firefly which is attracted to another more attractive or the $j$ th firefly is determined by the equation of $x_{i+1}=x_{i}+\beta_{0} e^{-\gamma r_{i j}^{2}}\left(x_{j}-x_{i}\right)+\alpha($ rand $1 / 2)$, where the second term is from the attraction and the third term is the randomisation of movement with $\alpha$ being the randomisation parameter and rand is a random number with uniform distribution in the $[0,1]$ interval or a normal distribution $N(0,1)$. In the $d$ dimensions it is possible to use the randomisation term to modify the parameters with significantly different scales. This term is useful to explore the search space with an increased diversity of the solutions. This movement equation is then a fine balance between local intensive exploitation and global exploration.

3.5. Sequential Procedures of the Proposed IMSM. The sequential optimisation method based on simplex designs firstly introduced by Spendley et al. expresses contrary to fundamental factorial designs in some characteristics [19]. This rigid simplex method (RS) is described as an evolutionary method for multiple parameter and interactive optimisation systems. It can find the global optimum with fewer trials via empirical, self-improving, and efficient strategies. The RS is based on geometry of the convex hull with an initial $k+1$ design points, where $k$ is the number of parameters that correspond with dimensions of searching space. Thus, in case of two-parameter optimisation, the simplex design takes the form of a triangle and in case of three parameters, it will be a tetrahedron. After evaluating and ranking the responses from all initial design points, the RS procedure is sequential with a removal of the least favourable response design point from an initial simplex. Continually forming a new simplex, one opposite new design point is determined in the hyperplane of the remaining design points. The RS evolves toward the optimum by simultaneously improving all parameters in every vertex. In order to improve the performance of the RS, many modifications have been proposed. One among them, the Nelder-Mead or modified simplex method (MSM), offers the effectiveness of the rapid convergence by employing an expansion or a contraction operator of the reflection according to quality of the responses including the massive contraction toward the best vertex. For possible stopping criterions Nelder and Mead included the standard deviation of the estimated responses at all the vertices of the simplex and the parameter levels and compared with their specified tolerances [20]. However, in this research there are some modifications applied to the original MSM.

In the IMSM, when focusing on the two-parameter maximisation of the composite desirability function level $(D)$

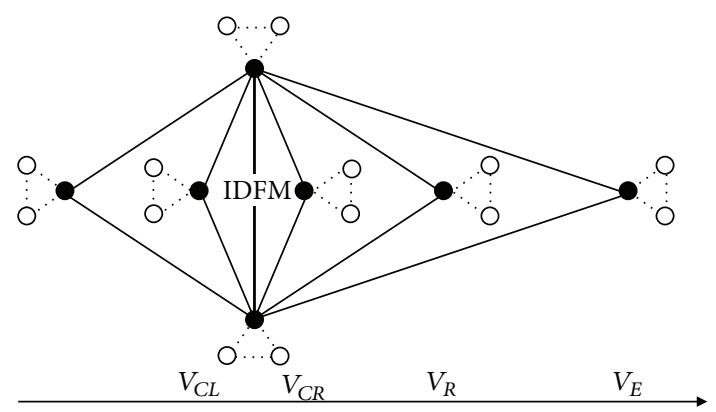

FIGURE 2: Different simplex moves of $V_{R}, V_{E}, V_{C R}, V_{C L}$, and the IDFM from the rejected trial vertex $\left(V_{L}\right)$.

or $D$ response with a triangle simplex, sequential procedures are given as follows. The simplex design is first applied at either arbitrary design points or previous operating design points within the safe region of operation including the neighbourhood design points from artificial intelligence mechanisms from the HSA and FA (Figure 2). All three design points evaluated their own $D$ responses and the best will be selected to form a simplex. All selected design points evaluated their composite desirability function levels and are categorised as the vertices corresponding to the highest $\left(V_{H}\right)$, second lowest $\left(V_{S L}\right)$, and lowest $\left(V_{L}\right)$ of $D$ responses. The lowest $D$ response vertex is identified and reflected in the opposite hyperface to obtain the reflected vertex $\left(V_{R}\right)$ via the centroid $(\bar{P})$ obtained by all remaining vertices in the simplex. Hence the reflected vertex $\left(V_{R}\right)$ may be given by

$$
V_{R}=\bar{P}+\alpha\left(\bar{P}-V_{L}\right)
$$

where $\bar{P}$ is the centroid of the design points on the reflecting line. In general, the reflection coefficient parameter of $\alpha$ is greater than 0 and it is the ratio of the distance between $\bar{P}-V_{L}$ and $\bar{P}-V_{R}$ and is normally defined to be 1 . If the $D$ response of $V_{R}$ lies between the lowest and highest levels, a new simplex is restarted by replacing the vertex $V_{L}$ with $V_{R}$. If the response of $V_{R}$ produces the highest level from the new simplex, an expansion operation is applied so as to further enhance the $D$ response level by expanding the reflected vertex $V_{R}$ along the direction of $\bar{P}-V_{R}$ to a new expansion vertex of $V_{E}$ via the following function:

$$
V_{E}=\bar{P}+\gamma\left(\bar{P}-V_{L}\right)
$$

where the expansion coefficient parameter of $\gamma$ is the ratio of the distance between $\bar{P}-V_{E}$ and $\bar{P}-V_{R}$ and is normally defined to be larger than 1. If the expansion vertex of $V_{E}$ produces the highest level of $D$ response when compared to $D$ responses from others, the new simplex is restarted by replacing $V_{H}$ with $V_{E}$. Otherwise, the simplex is restarted by replacing $V_{H}$ with $V_{R}$. In some situation of the IMSM there is a use of two contraction processes. Firstly, if the reflected vertex of $V_{R}$ produces the second highest level of the $D$ response only with the exception of $V_{L}$, a positive contraction process may 


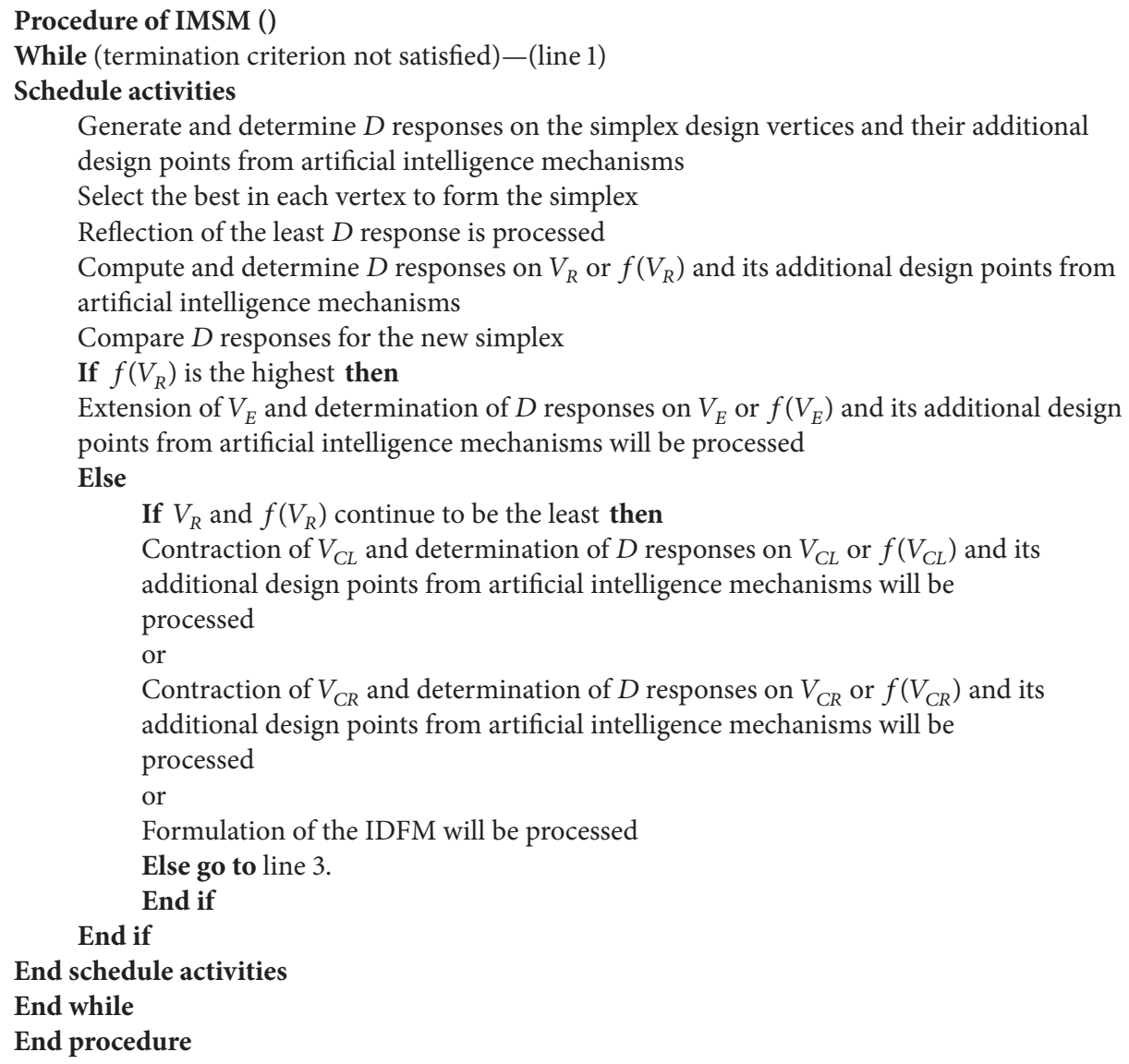

Pseudocode 1: Pseudocode of IMSM.

be made to generate the contracted vertex of $V_{C R}$ using the following equation:

$$
V_{C R}=\bar{P}+\beta^{+}\left(\bar{P}-V_{L}\right) \text {, }
$$

where $\beta^{+}$is the positive contraction coefficient parameter with the feasible range of $[0,1)$. If the reflected vertex of $V_{R}$ produces the lowest level of the $D$ response of all design points, the negative contraction process may be made to generate the contracted vertex of $V_{C L}$ using the following equation:

$$
V_{C L}=\bar{P}-\beta^{-}\left(\bar{P}-V_{L}\right),
$$

where $\beta^{-}$is the negative contraction coefficient parameter with the feasible range of $[0,1)$. It is noticed that these two contraction processes may be achieved within the unfavourable $D$ responses for a decision maker. However, in this research there is a use of the IDFA via the IDFM based on all $2(k+3)$ design points instead of the massive contraction or a shrink process to give an additional flexibility. The next run is carried out with parameters set at values corresponding to this new design point. An idea of IMSM's logical decision and its flowchart are shown in Pseudocode 1 and Figure 3.

\section{Numerical Results and Discussions}

The objective of a surface lapping process (SLP) is to remove material with a smooth and flat surface with dimensionally accurate specimens to high tolerance. Various parameters from the SLP can vary from application such as a lapping machine with different levels of speed and lapping plates, workstations for controlling lapping fixtures and the equipment to control the plate flatness. During the preliminary process parameter analyses, the brainstorming activities among all engineers from departments of process, advanced process development, product, tooling, and quality assurance announced eight possible parameters affecting the process responses of interest. They consist of lapping time, lapping speed, lapping pressure, $\mathrm{Al}_{2} \mathrm{O}_{3}$ charging pressure, type of lapping plate, aluminum oxide stone grit size, cutting fluid, and the number of experimental specimens per fixture. However, in this research the type of lapping plate, the cutting fluid, the aluminum oxide stone grit size, and the specimens per fixture are controlled with the $20 \mu \mathrm{m}$ lapping plate of cubic boron nitride (CBN) with the hexagonal tiles, the alpha2 fluid type, the 220 grit size of aluminum oxide stone, and 22 pieces, respectively. The feasible levels, the current operating condition, their possible differences of operation $(\Delta)$, and types of all influential parameters of the lapping time $\left(x_{1}\right)$, 


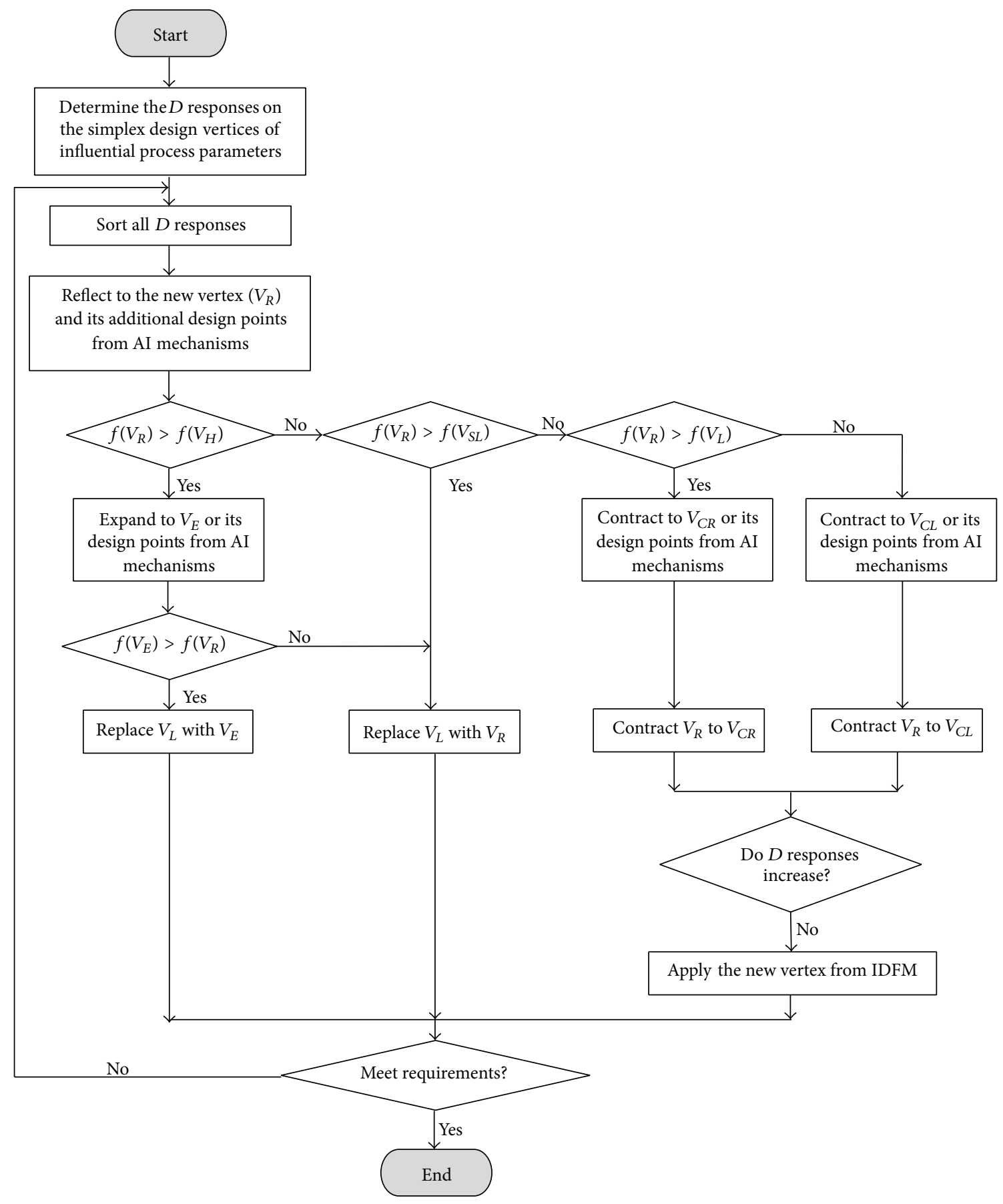

FIGURE 3: Flowchart of IMSM.

lapping speed $\left(x_{2}\right)$, downward pressure $\left(x_{3}\right)$, and charging pressure $\left(x_{4}\right)$ are shown in Table 3.

The purpose of the problem is to improve the SLP of the disk clamps on three different responses by controlling four parameters. Three major SLP's quality performance measures consist of the material removal $\left(y_{1}\right)$, lap width $\left(y_{2}\right)$, and clamp force $\left(y_{3}\right)$ as shown in Figure 4 . In addition, the material removal is based on the process requirement specification whereas the remaining responses of lap width and clamp force are measured and depend on customer requirement specifications. The process and customer specifications are required to reach the nominal value. Currently, it is found that the process quality characteristics are still quite low at 0.4554 of the composite desirability function level or $D$ response. A measure of the sample mean and standard deviation of the initial and finished height is done before and after the lapping process via a free-state height gage from Mitutoyo. The volume removed of materials is then calculated via the multiplication of the sectional area and the mean diameter of specimens. The sample mean and standard 

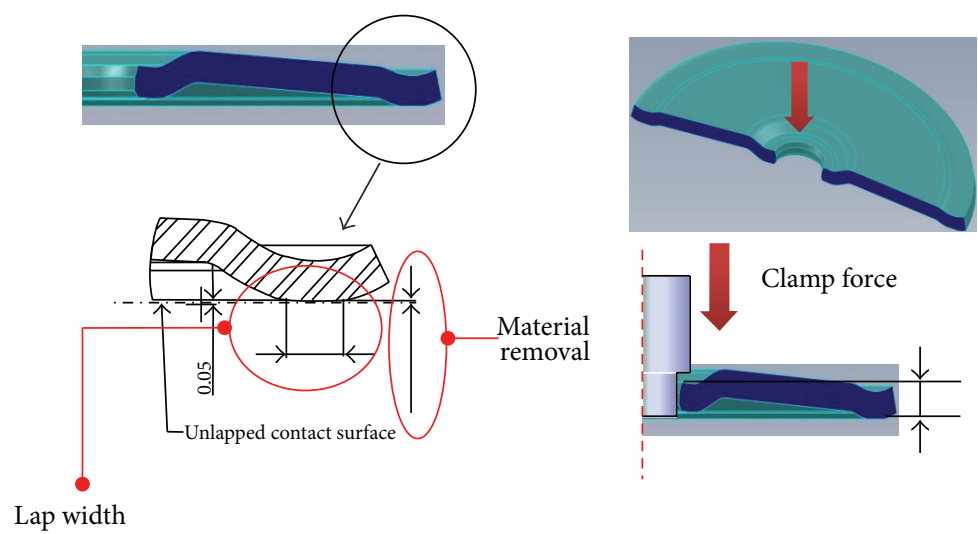

Figure 4: Measurements of $y_{1}, y_{2}$, and $y_{3}$ for the SLP.

TABle 3: Process parameters and their feasible and current levels.

\begin{tabular}{lccccc}
\hline \multirow{2}{*}{ Process parameter } & \multicolumn{5}{c}{ Feasible levels } \\
& Lower & Upper & Current & $\Delta$ & Unit \\
\hline$x_{1}$ & 30 & 999 & 60 & 1.0 & sec. \\
$x_{2}$ & 20 & 80 & 30 & 1.0 & rpm \\
$x_{3}$ & 4 & 15 & 8 & 0.25 & psi \\
$x_{4}$ & 3 & 15 & 8 & 0.25 & psi \\
\hline
\end{tabular}

deviation of the lap width and clamp force are measured via an OGP smartscope CNC vision model: ZIP 250 with DRS 500 laser and Instron 3344 force tester, respectively. Analyses of the test methods and measuring instruments including the entire processes to obtain measurements are considered to determine the uncertainty of our measurement systems. In this research, they were done before performing an improvement of the manufacturing process via \% GR\&R and $P / T$ ratio to address the precision of a measurement system and the ratio of the measurement system precision to the total tolerance of the manufacturing process, respectively. From additional tests, the levels of $P / T$ ratio and $\%$ GR $\&$ R are within the acceptable ranges as shown in Table 4.

In screening experiments a completely randomised design or one-way analysis of variance (ANOVA) is used to analyse all process parameters. The past experimental data can summarised the significant parameters categorised by the natural and $D$ responses. If $P$ value exceeds the $5 \%$ preset value of significance level $(\alpha)$, there is no effect of parameters. In accordance with the experimenter experience, all SLP parameters except $x_{4}$ are being confirmed for their statistically significant effects on all actual and desirability function responses as appeared in Table 5. However, in this investigation, the charging pressure affects the lap width and its desirability function as well.

On the IMSM, four SLP parameters have significant effects on all the responses from the preliminary experiments carried out using the available operating conditions of the company. Though the mean and standard deviation of actual responses of material removal, lap width and clamp force including its Ppk indicate a good reproducibility of the current condition of $\left(x_{1}, x_{2}, x_{3}\right.$, and $\left.x_{4}\right)$ at $(60,30$,
8.0 and 8.0) The design points of the initial simplex consist of five vertices. In each vertex the additional two design points are also established via metaheuristic mechanisms of the HSA and FA. The corresponding $D$ responses in the $i$ th vertex and its metaheuristic design points $\left(V_{i \mathrm{HSA}}\right.$ and $\left.V_{i \mathrm{FA}}\right)$ are determined and the best one will be included in the simplex for the next sequence of the IMSM. The assigned values of the HSA and FA parameters are suggested by the experts of the company and decided by the recommended levels from the literatures. All design points that are carried out according to their corresponding responses are measured in forms of the $D$ response. They are categorised in descending orders of $V_{H}, V_{S 3}, V_{S 2}, V_{S 1}$, and $V_{L}$. The simplex is then moved in the direction given by the rules of the IMSM including reflection, expansion, and contraction vertices including the interactive desirability function model (IDFM). The experimental conditions from the proposed IMSM based on the flowchart for all the vertices and variations of the parameter levels throughout the multiple response surface optimisation are given in Table 6.

The SLP evolution with four cycles via the IMSM can be described as follows. After ranking all vertices in the initial simplex from the worst vertex with the $D$ response of 0.5829 to the best vertex with the $D$ response of 0.8729 , the worst vertex was reflected to the opposite face. The new vertex $\left(V_{6}\right)$ including its neighbourhood design points from the HSA $\left(V_{6 \mathrm{HSA}}\right)$ and the FA $\left(V_{6 \mathrm{FA}}\right)$ were generated and determine their $D$ responses in order to establish the new simplex. During the reflection procedure the experimental domains for all parameters were justified within possible operating levels throughout. When the best reflected vertex was carried out, the $D$ response was worse than the remaining vertices. This result indicated that the $V_{C L}$ including its neighbourhood design points from the HSA $\left(V_{7 \mathrm{HSA}}\right)$ and the FA $\left(V_{7 \mathrm{FA}}\right)$ could be generated to form the new simplex. After determining the best of $D$ responses this led to the worst, the first interactive desirability function model (IDFM ${ }^{1}$ ) was formulated via all previous design points.

In analysing the experimental data for the $\mathrm{IDFM}^{1}$, the goodness-of-fit checking for the regression equation or the model is necessarily required. These model adequacy checking tests categorised by the actual response includes test 
TABLE 4: Measurement system analysis.

\begin{tabular}{|c|c|c|c|}
\hline Response & Device & $P / T$ ratio & $\%$ GR\&R \\
\hline Material removal & Free state height gage & $8.0 \%$ & $5.33 \%$ \\
\hline Lap width & OGP smartscope CNC vision model: ZIP 250 with DRS 500 laser & $1.95 \%$ & $1.4 \%$ \\
\hline Clamp force & Instron 3344 force tester & $15.1 \%$ & $2.28 \%$ \\
\hline
\end{tabular}

TABLE 5: One-way ANOVA: process parameters versus actual and desirability function responses.

\begin{tabular}{|c|c|c|c|c|c|c|c|}
\hline \multirow{3}{*}{ Parameter } & \multicolumn{7}{|c|}{$P$ value } \\
\hline & \multicolumn{3}{|c|}{ Actual response } & \multicolumn{4}{|c|}{ Desirability function response } \\
\hline & $y_{1}$ & $y_{2}$ & $y_{3}$ & $d_{1}\left(\widehat{y}_{1}\right)$ & $d_{2}\left(\widehat{y}_{2}\right)$ & $d_{3}\left(\hat{y}_{3}\right)$ & $D$ \\
\hline$x_{1}$ & 0.000 & 0.000 & 0.000 & 0.000 & 0.000 & 0.000 & 0.000 \\
\hline$x_{2}$ & 0.000 & 0.000 & 0.000 & 0.000 & 0.000 & 0.000 & 0.000 \\
\hline$x_{3}$ & 0.000 & 0.000 & 0.000 & 0.000 & 0.000 & 0.000 & 0.000 \\
\hline$x_{4}$ & 0.096 & 0.018 & 0.429 & 0.100 & 0.020 & 0.429 & 0.077 \\
\hline
\end{tabular}

for significance of the regression model, test for significance on the IDFM ${ }^{1}$ coefficients, and test for lack of fit via an analysis of variance or ANOVA (Table 7). On analyses of the estimated responses of the material removal $\left(\widehat{y}_{1}\right)$, lap width $\left(\hat{y}_{2}\right)$, and clamp force $\left(\hat{y}_{3}\right)$ it is recommended that the linear regression models are statistically significant for all responses. The associated $P$ values for the models of $\hat{y}_{1}, \widehat{y}_{2}$, and $\widehat{y}_{3}$ in the form of ANOVA are less than 0.05 with the 95\% confidence except for $x_{3}$ for $\widehat{y}_{3}$. These express that the linear regression models provide good explanations of the relationship between the process parameters and all types of responses. The response equations are given as follows:

$$
\begin{aligned}
\widehat{y}_{1}= & 1.27057+0.18951 x_{1}+0.12805 x_{2}+0.07607 x_{3} \\
& +0.05757 x_{4}, \\
\widehat{y}_{2}= & 0.75331+0.038274 x_{1}+0.027153 x_{2}+0.016471 x_{3} \\
& +0.012711 x_{4}, \\
\widehat{y}_{3}= & 26.8428-0.14695 x_{1}-0.0769 x_{2}-0.05294 x_{3} \\
& -0.0403904 x_{4} .
\end{aligned}
$$

The actual responses are then transformed into the scale free or desirability function response of the-nominal-thebest for the material removal, lap width, and clamp force denoted as $d_{1}\left(\widehat{y}_{1}\right), d_{2}\left(\widehat{y}_{2}\right)$, and $d_{3}\left(\widehat{y}_{3}\right)$, respectively. It is the level between 0 and 1 and there is an increase when the corresponding response level becomes more desirable. The desirability functions based upon the initial shape, bound, and target are generated for all responses (Figure 5). The associated levels for all DFA transformation and IDFM parameters are given in Tables 8 and 9, respectively. The optimal solution, the $\operatorname{IDFM}^{1}$ of $\left(x_{1}, x_{2}, x_{3}\right.$, and $\left.x_{4}\right)$ at $(40,33$, 8.0, and 8.0) or $V_{8}$, was solved via the optimisation model. From $V_{8}$ and its neighbourhood design points, the corresponding desirability function levels of $d_{1}\left(\widehat{y}_{1}\right), d_{2}\left(\widehat{y}_{2}\right), d_{3}\left(\widehat{y}_{3}\right)$, and $D$ were $1.0000,0.8842,0.7615$, and 0.8762 , respectively. The associated result of $\widehat{y}_{1}$ was considered satisfactory, but the estimated actual responses of $\hat{y}_{2}$ and $\hat{y}_{3}$ were not. Thus, the sequential procedures of the IMSM were continued by forming the new simplex.

With the basic simplex rules each vertex with the best $D$ response from all three possible deign points was accumulated to form the new simplex. The reflected rule was then applied to calculate the new vertex $\left(V_{9}\right)$ including its neighbourhood design points from the HSA $\left(V_{9 \mathrm{HSA}}\right)$ and the FA $\left(V_{9 \mathrm{FA}}\right)$ design points. The resulting $D$ response was 0.7277 . This result indicated that the $V_{C L}$ including its neighbourhood design points from the HSA $\left(V_{10 \mathrm{HSA}}\right)$ and the FA $\left(V_{10 \mathrm{FA}}\right)$ formed the new simplex. However, the $D$ response deteriorated and there was a use of the second interactive desirability function model $\left(\mathrm{IDFM}^{2}\right)$. After team brainstorming the decision maker decided to adjust the parameter bounds as appeared in Table 9. The optimal solution, the IDFM $^{2}$ of $\left(x_{1}, x_{2}, x_{3}\right.$, and $\left.x_{4}\right)$ at $(37,40,7.0$, and 5.0) or $V_{11}$, was solved via the optimisation model. From $V_{11}$ and its neighbourhood design points, the corresponding desirability function levels of $d_{1}\left(\widehat{y}_{1}\right), d_{2}\left(\widehat{y}_{2}\right), d_{3}\left(\hat{y}_{3}\right)$, and $D$ were 1.0000 , $1.0000,0.7652$, and 0.9146 , respectively.

The procedures were repeated to achieve the new reflected vertex of $V_{12}$, but the outcome was not preferable. The positive contraction process to the reflected vertex or $V_{C R}$ $\left(V_{13}\right)$ was then carried out. However, the $D$ response was not satisfactory and there was a use of the third interactive desirability function model (IDFM ${ }^{3}$ ) with the new levels of power coefficients as shown in Table 9 and Figure 6. The optimal solution, the $\operatorname{IDFM}^{3}$ of $\left(x_{1}, x_{2}, x_{3}\right.$, and $\left.x_{4}\right)$ at $(30,40,8.0$, and 5.0) or $V_{14}$, was solved via the optimisation model. From $V_{11}$ and its neighbourhood design points, the corresponding desirability function levels of $d_{1}\left(\hat{y}_{1}\right), d_{2}\left(\hat{y}_{2}\right), d_{3}\left(\hat{y}_{3}\right)$, and $D$ were $0.9958,1.0000,0.8924$, and 0.9572 , respectively. From Table 6, it was shown that the numerical results on the 4 th cycle repeated at the negative contraction process to the worst vertex or $V_{C L}\left(V_{16}\right)$ after realizing that the $V_{R}\left(V_{15}\right)$ led to the worse $D$ response when compared. There was then a use of the fourth interactive desirability function model $\left(\mathrm{IDFM}^{4}\right)$ with the shape parameters of $\left(\mathrm{PT}_{1}, \mathrm{PT} 2_{1}, \mathrm{PT}_{2}, \mathrm{PT}_{2}, \mathrm{PT}_{3}\right.$, and $\left.\mathrm{PT}_{3}\right)$ at $(0.1,0.1,1.0,1.0,0.3$, and 0.3$)$ as shown in 


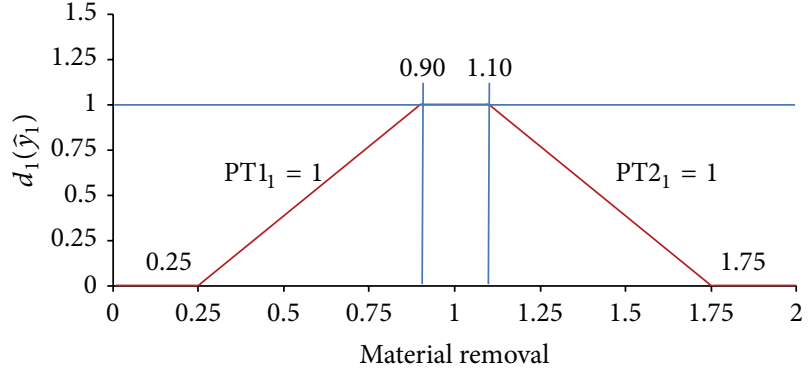

(a)

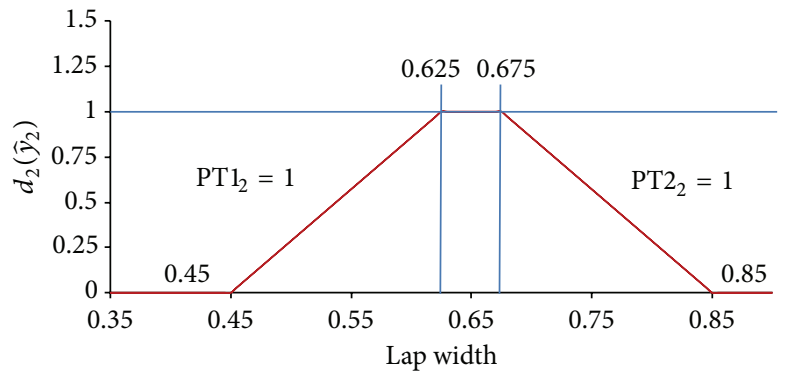

(b)

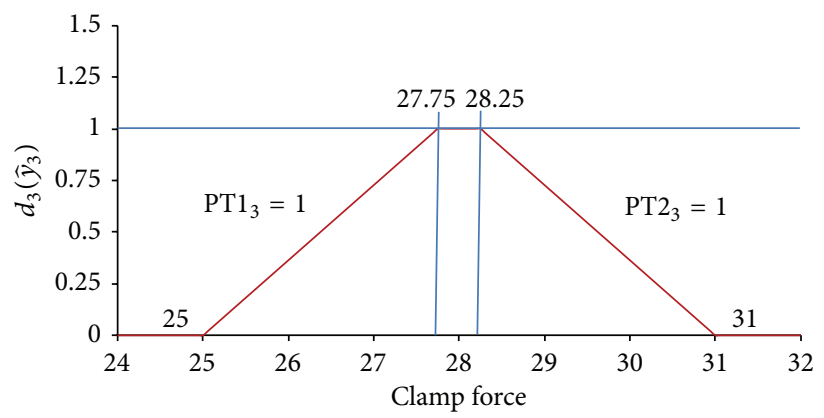

(c)

FIGURE 5: Power coefficients of the $\operatorname{IDFM}^{1}$ and IDFM ${ }^{2}$.

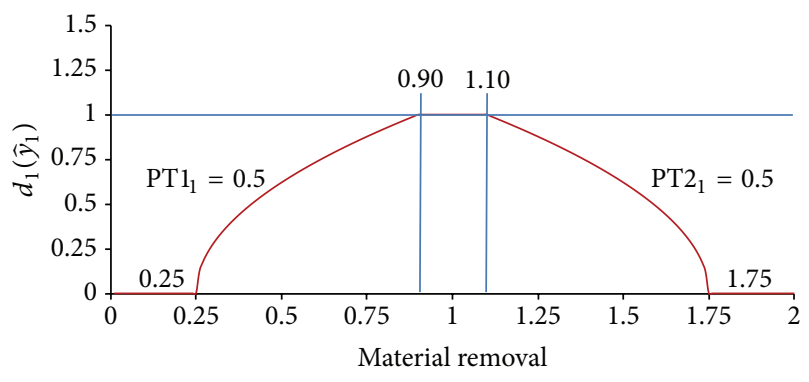

(a)

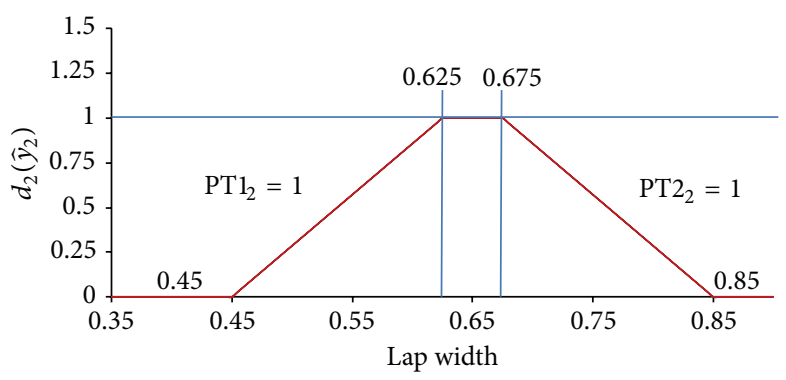

(b)

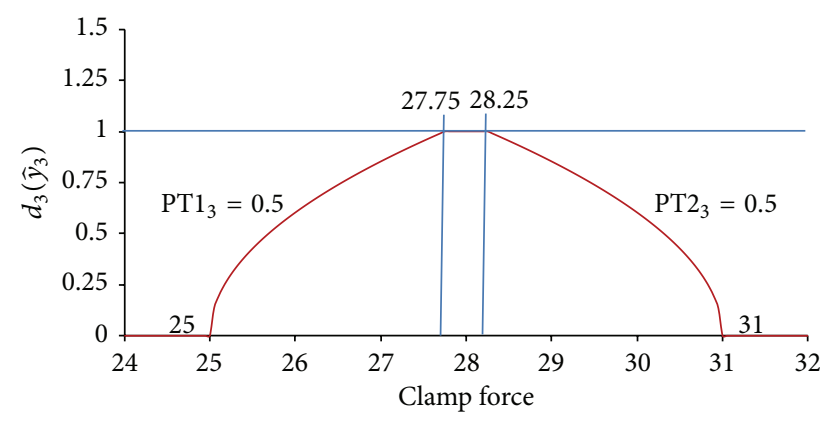

(c)

Figure 6: Power coefficients of the IDFM ${ }^{3}$.

Figure 7. The optimal solution, the $\operatorname{IDFM}^{4}$ of $\left(x_{1}, x_{2}, x_{3}\right.$, and $\left.x_{4}\right)$ was at $(33,35,9.0$, and 5.0) and the corresponding desirability function levels of $d_{1}\left(\hat{y}_{1}\right), d_{2}\left(\hat{y}_{2}\right), d_{3}\left(\hat{y}_{3}\right)$, and $D$ were $0.9853,1.0000,0.9435$, and 0.9734 , respectively. Within smaller number of cycles of the numerical results on the SLP the IMSM successfully found a satisfactory outcome of the $D$ response by adjusting shape parameters.

A confirmation technique via ANOVA is carried out to analyse experimental data in which the actual responses are evaluated under the current operating condition and the new 
TABLE 6: Experimental design points and their responses from the IMSM.

\begin{tabular}{|c|c|c|c|c|c|c|c|c|c|}
\hline Design point & $x_{1}$ & $x_{2}$ & $x_{3}$ & $x_{4}$ & $d_{1}\left(\widehat{y}_{1}\right)$ & $d_{2}\left(\hat{y}_{2}\right)$ & $d_{3}\left(\hat{y}_{3}\right)$ & $D$ & Rank \\
\hline $\begin{array}{l}V_{1} \\
V_{1 \mathrm{HSA}} \\
V_{1 \mathrm{FA}} \\
\end{array}$ & 48 & 26 & 7.00 & 7.50 & 0.9380 & 0.9444 & 0.7503 & 0.8729 & $V_{H}$ \\
\hline $\begin{array}{l}V_{2} \\
V_{2 \mathrm{HSA}} \\
V_{2 \mathrm{FA}} \\
\end{array}$ & 45 & 25 & 6.50 & 7.50 & 0.8093 & 0.9600 & 0.7464 & 0.8339 & $V_{S 3}$ \\
\hline $\begin{array}{l}V_{3} \\
V_{3 \mathrm{HSA}} \\
V_{3 \mathrm{FA}} \\
\end{array}$ & 52 & 27 & 7.50 & 7.50 & 0.9231 & 0.7250 & 0.7310 & 0.7880 & $V_{S 2}$ \\
\hline $\begin{array}{l}V_{4} \\
V_{4 \mathrm{HSA}} \\
V_{4 \mathrm{FA}} \\
\end{array}$ & 41 & 24 & 6.50 & 7.00 & 0.7043 & 0.7120 & 0.8254 & 0.7453 & $V_{S 1}$ \\
\hline $\begin{array}{l}V_{5} \\
V_{5 \mathrm{HSA}} \\
V_{5 \mathrm{FA}} \\
\end{array}$ & 56 & 29 & 7.50 & 8.00 & 0.6297 & 0.4833 & 0.6508 & 0.5829 & $V_{L}$ \\
\hline $\begin{array}{l}V_{6} \\
V_{6 \mathrm{HSA}} \\
V_{6 \mathrm{FA}} \\
\end{array}$ & 60 & 30 & 12.00 & 8.00 & 0.4969 & 0.4979 & 0.6202 & 0.5354 & $V_{R}$ \\
\hline $\begin{array}{l}V_{7} \\
V_{7 \mathrm{HSA}} \\
V_{7 \mathrm{FA}} \\
\end{array}$ & 60 & 40 & 8.00 & 8.00 & 0.6214 & 0.3212 & 0.7012 & 0.5192 & $V_{C L}$ \\
\hline$V_{8 I D F M}$ & 40 & 33 & 8.00 & 8.00 & 1.0000 & 0.8842 & 0.7615 & 0.8762 & IDFM $^{1}$ \\
\hline $\begin{array}{l}V_{9} \\
V_{9 \mathrm{HSA}} \\
V_{9 \mathrm{FA}} \\
\end{array}$ & 40 & 40 & 8.00 & 12.00 & 0.8711 & 0.6381 & 0.6933 & 0.7277 & $V_{R}$ \\
\hline $\begin{array}{l}V_{10} \\
V_{10 \mathrm{HSA}} \\
V_{10 \mathrm{FA}} \\
\end{array}$ & 40 & 40 & 12.00 & 8.00 & 0.8395 & 0.6602 & 0.7185 & 0.7357 & $V_{C L}$ \\
\hline$V_{\text {1IIDFM }}$ & 37 & 40 & 7.00 & 5.00 & 1.0000 & 1.0000 & 0.7652 & 0.9146 & $\mathrm{IDFM}^{2}$ \\
\hline $\begin{array}{l}V_{12} \\
V_{12 \mathrm{HSA}} \\
V_{12 \mathrm{FA}} \\
\end{array}$ & 42 & 24 & 6.6 & 7.1 & 0.7580 & 0.8800 & 0.7889 & 0.8073 & $V_{R}$ \\
\hline $\begin{array}{l}V_{13} \\
V_{13 \mathrm{HSA}} \\
V_{13 \mathrm{FA}} \\
\end{array}$ & 60 & 30 & 8 & 8 & 0.8395 & 0.7714 & 0.7105 & 0.7720 & $V_{C R}$ \\
\hline$V_{14 \mathrm{IDFM}}$ & 30 & 40 & 8.00 & 5.00 & 0.9958 & 1.0000 & 0.8924 & 0.9572 & IDFM $^{3}$ \\
\hline $\begin{array}{l}V_{15} \\
V_{15 \mathrm{HSA}} \\
V_{15 \mathrm{FA}} \\
\end{array}$ & 52 & 28 & 7.50 & 7.50 & 1.0000 & 0.7250 & 0.7310 & 0.8093 & $V_{R}$ \\
\hline $\begin{array}{l}V_{16} \\
V_{16 \mathrm{HSA}} \\
V_{16 \mathrm{FA}} \\
\end{array}$ & 41 & 24 & 6.50 & 7.00 & 0.7043 & 0.9070 & 0.8254 & 0.8079 & $V_{C L}$ \\
\hline$V_{\text {17IDFM }}$ & 33 & 35 & 9.00 & 5.00 & 0.9853 & 1.0000 & 0.9435 & 0.9734 & IDFM $^{4}$ \\
\hline
\end{tabular}

design point from the IMSM. It can also be seen that these experimental results on all scenario were statistically significant with $95 \%$ confidence interval with $P$ value of 0.000 . The numerical results suggested that the new one provided the better performance in terms of the statistical significance of $P$ value for all responses throughout. The desired or major quality characteristics on both process and customer specifications are improved and expressed in terms of sample mean, sample standard deviation, and process performance index as shown in Table 10. With this combination of process 
TABLE 7: Estimated effects and coefficients for the actual responses and their ANOVA for regression equation tables on the IDFM ${ }^{1}$.

\begin{tabular}{|c|c|c|c|c|c|c|c|}
\hline & onse & Constant & $x_{1}$ & $x_{2}$ & $x_{3}$ & $x_{4}$ & Regression ANOVA \\
\hline \multirow{3}{*}{$\widehat{y}_{1}$} & Coef. & 1.27057 & 0.18951 & 0.12805 & 0.07607 & 0.05757 & \multirow{3}{*}{0.000} \\
\hline & $T$ & 55.76 & 12.79 & 8.64 & 5.13 & 3.89 & \\
\hline & $P$ value & 0.000 & 0.000 & 0.000 & 0.001 & 0.001 & \\
\hline \multirow{3}{*}{$\widehat{y}_{2}$} & Coef. & 0.75331 & 0.038274 & 0.027153 & 0.016471 & 0.012711 & \multirow{3}{*}{0.000} \\
\hline & $T$ & 168.39 & 8.56 & 6.07 & 3.68 & 2.84 & \\
\hline & $P$ value & 0.000 & 0.000 & 0.000 & 0.001 & 0.008 & \\
\hline \multirow{3}{*}{$\widehat{y}_{3}$} & Coef. & 26.8428 & -0.14695 & -0.0769 & -0.05294 & -0.04039 & \multirow{3}{*}{0.000} \\
\hline & $T$ & 3.46 & -7.19 & -3.76 & -2.59 & -1.98 & \\
\hline & $P$ value & 0.000 & 0.000 & 0.001 & 0.015 & 0.058 & \\
\hline
\end{tabular}

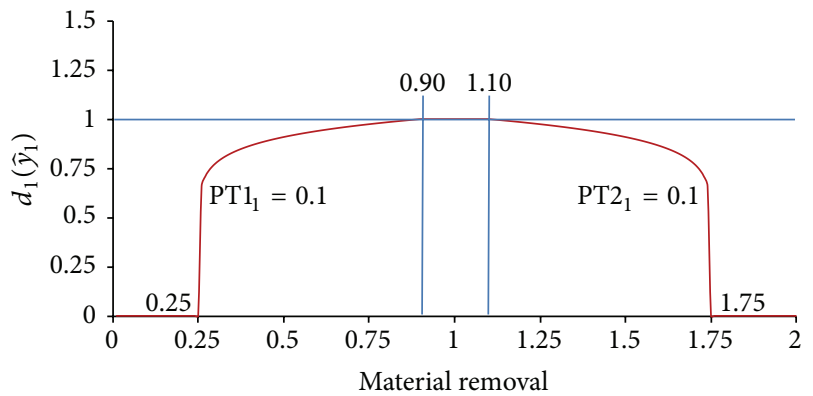

(a)

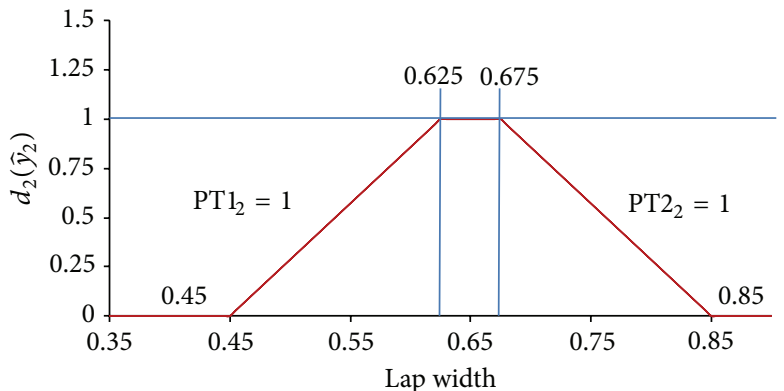

(b)

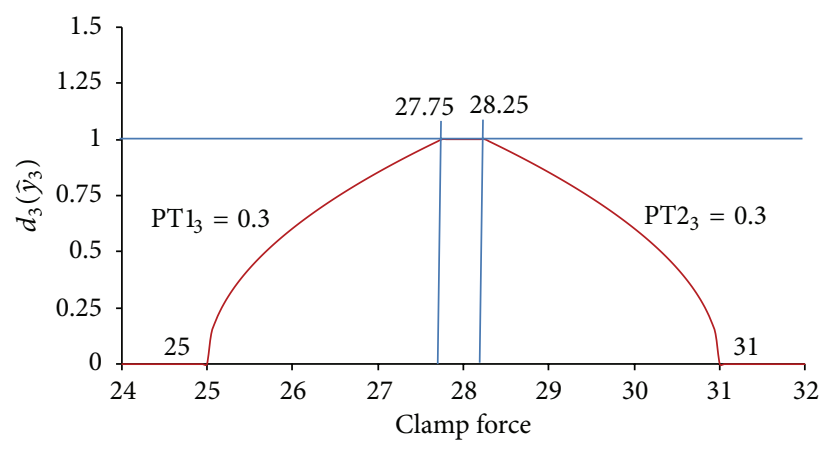

(c)

FIGURE 7: Power coefficients of the IDFM ${ }^{4}$.

parameters it leads to the successfully controlled surface quality characteristics of the work piece surface in contact with the disk with high manufacturing efficiency. The scenario of the higher levels of the process parameters of lapping speed $\left(x_{2}\right)$ and downward pressure $\left(x_{3}\right)$ indicates that there is constant and stable applied force on the specimen. Consequently, the minor surface quality measurement based on the flatness and the roughness is also improved as shown in Figures 8 and 9, respectively.

\section{Summary and Conclusion}

With the higher levels of demand of disk clamps in ultraprecision mechanical components, the surface lapping process needs to focus its major performances to meet process and customer satisfactions. This process intends to introduce a finely ground flat on disk clamps and removes material from
TABLE 8: Transformation DFA parameters and their levels.

\begin{tabular}{lcccc}
\hline \multirow{2}{*}{ Level for the $i$ th response } & \multicolumn{4}{c}{ DFA Parameter } \\
& $Y_{i}^{\mathrm{MIN}}$ & $Y_{i}^{\mathrm{MAX}}$ & $T_{i}^{\mathrm{MIN}}$ & $T_{i}^{\mathrm{MAX}}$ \\
\hline $\mathbf{1}$ & 0.25 & 1.75 & 0.90 & 1.10 \\
$\mathbf{2}$ & 0.50 & 0.8 & 0.625 & 0.675 \\
$\mathbf{3}$ & 25.0 & 28.0 & 27.75 & 28.25 \\
\hline
\end{tabular}

the disk contact radius to provide the desired dimension of the surface. The parameters of the lapping time, lapping speed, downward pressure, and charging pressure have an important role from the process knowledge when compared to other parameters of the type of lapping plate, aluminum oxide stone grit size, cutting fluid, and the number of experimental specimens per fixture. Consequently, they are controlled during the optimisation phase. However, it is 


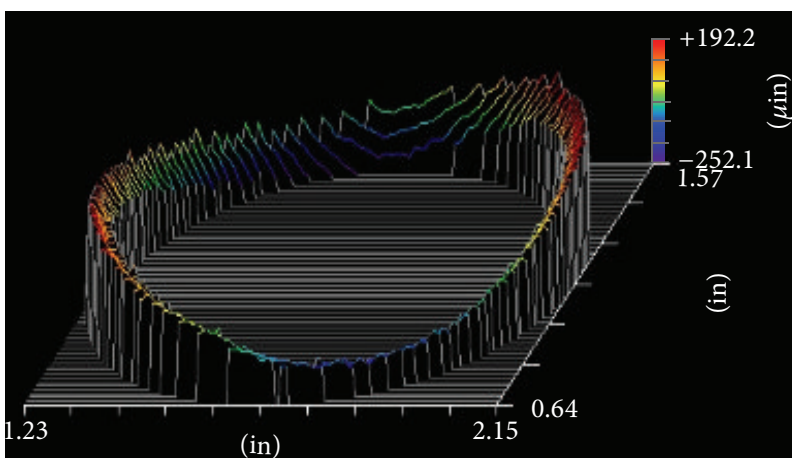

CUR

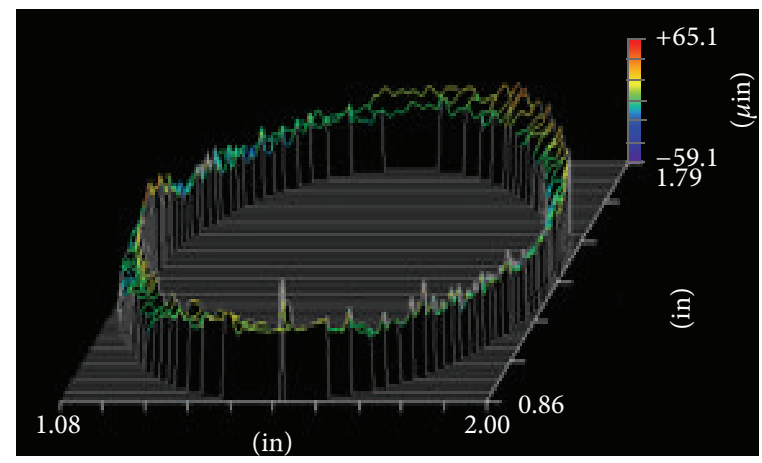

NEW

FIGURE 8: Comparative results of the flatness based on the current (CUR) and the new (NEW) operating conditions.
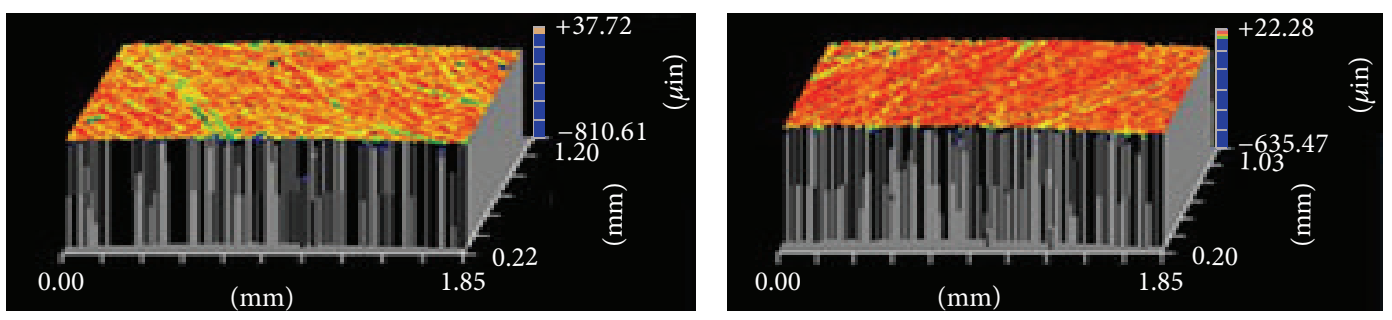

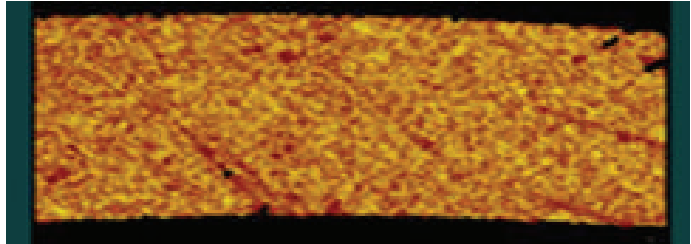

CUR

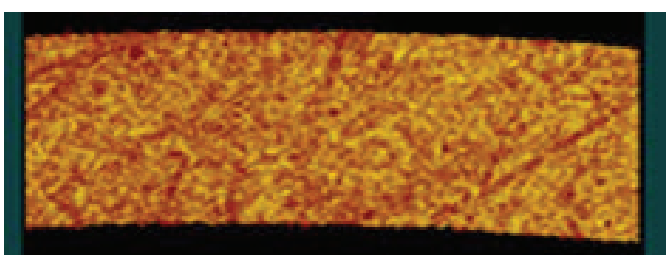

NEW

FIGURE 9: Comparative results of the roughness based on the current (CUR) and the new (NEW) operating conditions.

TABLE 9: Lower and upper bounds of process parameters and power coefficients of the IDFM for all iterations.

\begin{tabular}{|c|c|c|c|c|c|c|}
\hline \multirow{2}{*}{ Parameter } & & & \multicolumn{4}{|c|}{ Cycle $(n)$} \\
\hline & & & 1 & 2 & 3 & 4 \\
\hline \multirow{8}{*}{ Process parameter $\left(x_{j}\right)$} & \multirow{2}{*}{$x_{1}$} & $\mathrm{LB}_{j}$ & 40 & 30 & 30 & 30 \\
\hline & & $\mathrm{UB}_{j}$ & 60 & 60 & 60 & 60 \\
\hline & \multirow{2}{*}{$x_{2}$} & $\mathrm{LB}_{j}$ & 30 & 30 & 30 & 30 \\
\hline & & $\mathrm{UB}_{j}$ & 40 & 40 & 40 & 40 \\
\hline & \multirow{2}{*}{$x_{3}$} & $\mathrm{LB}_{j}$ & 8.0 & 5.0 & 5.0 & 5.0 \\
\hline & & $\mathrm{UB}_{j}$ & 12.0 & 15.0 & 15.0 & 15.0 \\
\hline & \multirow{2}{*}{$x_{4}$} & $\mathrm{LB}_{j}$ & 8.0 & 5.0 & 5.0 & 5.0 \\
\hline & & $\mathrm{UB}_{j}$ & 12.0 & 15.0 & 15.0 & 15.0 \\
\hline \multirow{6}{*}{ IDFM parameter for $y_{i}$} & \multirow{2}{*}{$y_{1}$} & $\mathrm{PT}_{i}$ & 1.0 & 1.0 & 0.5 & 0.1 \\
\hline & & $\mathrm{PT} 2_{i}$ & 1.0 & 1.0 & 0.5 & 0.1 \\
\hline & \multirow{2}{*}{$y_{2}$} & $\mathrm{PT}_{i}$ & 1.0 & 1.0 & 1.0 & 1.0 \\
\hline & & $\mathrm{PT} 2_{i}$ & 1.0 & 1.0 & 1.0 & 1.0 \\
\hline & \multirow{2}{*}{$y_{3}$} & $\mathrm{PT}_{i}$ & 1.0 & 1.0 & 0.5 & 0.3 \\
\hline & & $\mathrm{PT} 2_{i}$ & 1.0 & 1.0 & 0.5 & 0.3 \\
\hline
\end{tabular}

difficult to optimise this process due to multiple responses to be considered and they consist of the material removal, lap width, and clamp force. Therefore, the optimisation of the surface lapping process or SPL is challenge to the existing ultraprecision technologies. With the multiple optimisation all interesting responses are considered in order to achieve their targets. The desirability function approach in the case of the-nominal-the-best with various shape parameters was applied to compromise those actual responses in terms of the compromise desirability function level or $D$ response. The sequential modified simplex method then merges with the interactive desirability function model (IDFM) instead of the massive contraction and it is called the interactive modified simplex method (IMSM).

The IMSM is used to optimise online the parameter levels of the SLP in order to simultaneously optimise all actual responses or maximise the $D$ response. An additional contribution of this study was the development of artificial intelligence mechanisms of the harmony search and firefly algorithms on all vertices of the simplex design points to search the possible better outcome. An aim is to avoid getting the unsatisfactory $D$ responses to continue the sequential 
TABLE 10: Desired or major quality characteristics at the current (CUR) and new (NEW) operating condition.

\begin{tabular}{llllrrr}
\hline \multirow{2}{*}{ Major quality characteristics } & \multicolumn{2}{c}{ Sample mean } & \multicolumn{2}{c}{ Sample standard deviation } & \multicolumn{2}{c}{ Ppk } \\
& CUR & NEW & CUR & NEW & CUR & NEW \\
\hline Material removal & 1.383 & 0.760 & 0.134 & 0.097 & 0.91 \\
Lap width & 0.767 & 0.635 & 0.028 & 0.035 & 0.01 \\
Clamp force & 26.54 & 27.28 & 0.585 & 0.465 & 1.41 \\
\hline
\end{tabular}

procedures of the conventional MSM such as the reflection, the contraction to the reflected, or the worst vertex. To evaluate the performance of the IMSM, numerical experiments on the SLP were presented within four cycles. From the DM and his team, there were bound adjustment, tightening, and relaxation modes based on the shape, bound, and target of the IDFM for the IMSM. The experimental results showed that according to the highest level of the $D$ response of 0.9734 , the preferable levels of process parameters of lapping time, lapping speed, downward pressure, and charging pressure from the fourth cycle of the IDFM were 33, 35, 9.0, and 5.0, respectively.

As expected, there is a level decrease of process parameters of the lapping time and charging pressure in the course of the IMSM evolution. Indeed the IMSM provides the lower levels of the lapping time, higher levels of lapping speed, and slightly higher levels of downward pressure in order to compromise all the responses by decreasing the material removal and increasing the lap width and clamp force. The second reason is that these levels have an additional influence on the better surface quality. Lower levels of charging pressure are necessary to avoid the material removal drifting. The material removal can meet the process specification with the levels of mean, standard deviation, and Ppk at 0.760, 0.097, and 2.01, respectively. This operating condition achieved the proper levels of the lap width dimension with the levels of mean, standard deviation, and Ppk at $0.635,0.035$, and 1.41 , respectively, and clamp force with the levels of mean, standard deviation, and Ppk at 27.28, 0.465, and 1.58, respectively, when compared to the customer specification. These experimental results allow us to conclude that the interactive modified simplex method with desirability function and artificial intelligence mechanisms is a suitable method for online optimisation in the surface lapping process. It may be easily applied to drive other related processes toward the optimal operating conditions.

In conjunction with the modified simplex method the interactive desirability function model (IDFM) allows the decision maker and his team to apply whenever there is deterioration in the composite desirability function level or $D$ response instead of the conventional massive contraction. They could also adjust any desirability function parameters such as the shape, bound, and target in the preference articulation process (Jeong and Kim, 2009). The IDFM can be effective in providing alternatives of compromise design points that is faithful to the preference structure. Moreover, metaheuristic mechanisms of the harmony search and firefly algorithms are also adapted to this interactive modified simplex method before forming the simplex. Other applications of the optimised parameter levels or other classes of artificial intelligence techniques to the IMSM are left for future study. Although the proposed modified simplex method can provide the enhancement during the multiple response surface optimisation, it could be expected that the fruitful results will be obtained by combining another family of sequential procedures based on the simplex designs in forms of new evolutionary methods.

\section{Conflict of Interests}

The authors declare that there is no conflict of interests regarding the publication of this paper.

\section{Acknowledgments}

This work was supported by the Higher Education Research Promotion and National Research University Project of Thailand, Office of Higher Education Commission.

\section{References}

[1] J. W. Cha, S. C. Hwang, and E. S. Lee, "Evaluation of $\mathrm{Y}_{2} \mathrm{O}_{3}$ surface machinability using ultra-precision lapping process with IED," Journal of Mechanical Science and Technology, vol. 23, no. 4, pp. 1194-1201, 2009.

[2] Z. Qiu, F. Z. Fang, L. Ding, and Q. Zhao, "Investigation of diamond cutting tool lapping system based on on-machine image measurement," The International Journal of Advanced Manufacturing Technology, vol. 56, pp. 79-86, 2011.

[3] I. D. Marinescu, E. Uhlmann, and T. K. Doi, Handbook of Lapping and Polishing, Taylor \& Francis, 2007.

[4] J.-D. Kim and M.-S. Choi, "A study on the optimization of the cylindrical lapping process for engineering fine-ceramics $\left(\mathrm{Al}_{2} \mathrm{O}_{3}\right)$ by the statistical design method," Journal of Materials Processing Technology, vol. 52, no. 2-4, pp. 368-385, 1995.

[5] H. L. Lin, "Optimizing the auto-brazing process quality of aluminum pipe and flange via a Taguchi-Neural-Genetic approach," Journal of Intelligent Manufacturing, vol. 23, no. 3, pp. 679-686, 2012.

[6] H. L. Lin, "The use of the Taguchi method with grey relational analysis and a neural network to optimize a novel GMA welding process," Journal of Intelligent Manufacturing, vol. 23, no. 5, pp. 1671-1680, 2012.

[7] T.-R. Lin, "A comparative study between smaller-the-better and normal-the-best quality characteristics when lapping ceramic blocks," International Journal of Advanced Manufacturing Technology, vol. 31, no. 1-2, pp. 155-163, 2006.

[8] Y. Zhang, I. D. Marinescu, and R. Vandenboom, "Optimisation of D2 steel lapping with a polymer plate," International Journal of Abrasive Technology, vol. 3, no. 3, pp. 203-214, 2010. 
[9] R. Ramezanian and M. Saidi-Mehrabad, "Hybrid simulated annealing and MIP-based heuristics for stochastic lot-sizing and scheduling problem in capacitated multi-stage production system," Applied Mathematical Modelling, vol. 37, no. 7, pp. 51345147, 2013.

[10] S. Salcedo-Sanz, D. Manjarrés, Á. Pastor-Sánchez, J. del Ser, J. A. Portilla-Figueras, and S. Gil-López, "One-way urban traffic reconfiguration using a multi-objective harmony search approach," Expert Systems with Applications, vol. 40, no. 9, pp. 3341-3350, 2013.

[11] T. V. Sibalija and V. D. Majstorovic, "An integrated approach to optimise parameter design of multi-response processes based on Taguchi method and artificial intelligence," Journal of Intelligent Manufacturing, vol. 23, no. 5, pp. 1511-1528, 2012.

[12] A. C. C. Carlos and L. B. Ricardo, "Evolutionary multiobjective optimization in materials science and engineering," Materials and Manufacturing Processes, vol. 24, no. 2, pp. 119-129, 2009.

[13] I. J. Jeong and K. J. Kim, "Stochastics and Statistics: an interactive desirability function method to multiresponse optimisation," European Journal of Operational Research, vol. 195, no. 2, pp. 412-426, 2009.

[14] A. Singh, S. Datta, S. S. Mahapatra, T. Singha, and G. Majumdar, "Optimization of bead geometry of submerged arc weld using fuzzy based desirability function approach," Journal of Intelligent Manufacturing, vol. 24, no. 1, pp. 35-44, 2013.

[15] K. S. Lee and Z. W. Geem, "A new meta-heuristic algorithm for continuous engineering optimization: harmony search theory and practice," Computer Methods in Applied Mechanics and Engineering, vol. 194, no. 36-38, pp. 3902-3933, 2005.

[16] E. Cuevas, "Block-matching algorithm based on harmony search optimisation for motion estimation," Applied Intelligence, vol. 39, no. 1, pp. 165-183, 2013.

[17] X. S. Yang, Nature-Inspired Metaheuristic Algorithms, Luniver Press, Beckington, UK, 2008.

[18] X.-S. Yang, "Firefly algorithms for multimodal optimization," in Stochastic Algorithms: Foundations and Applications, vol. 5792 of Lecture Notes in Computer Sciences, pp. 169-178, Springer, Berlin, Germany, 2009.

[19] M. T. Vakil Baghmisheh, M. Peimani, M. H. Sadeghi, M. M. Ettefagh, and A. F. Tabrizi, "A hybrid particle swarm-NelderMead optimization method for crack detection in cantilever beams," Applied Soft Computing Journal, vol. 12, no. 8, pp. 22172226, 2012.

[20] V. C. Mariani, L. G. Justi Luvizotto, F. A. Guerra, and L. Dos Santos Coelho, "A hybrid shuffled complex evolution approach based on differential evolution for unconstrained optimization," Applied Mathematics and Computation, vol. 217, no. 12, pp. 5822-5829, 2011. 


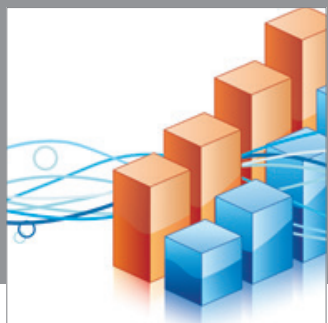

Advances in

Operations Research

mansans

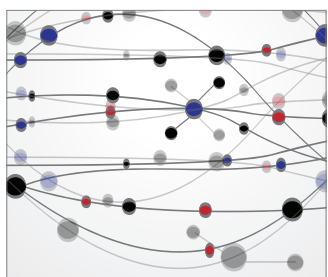

The Scientific World Journal
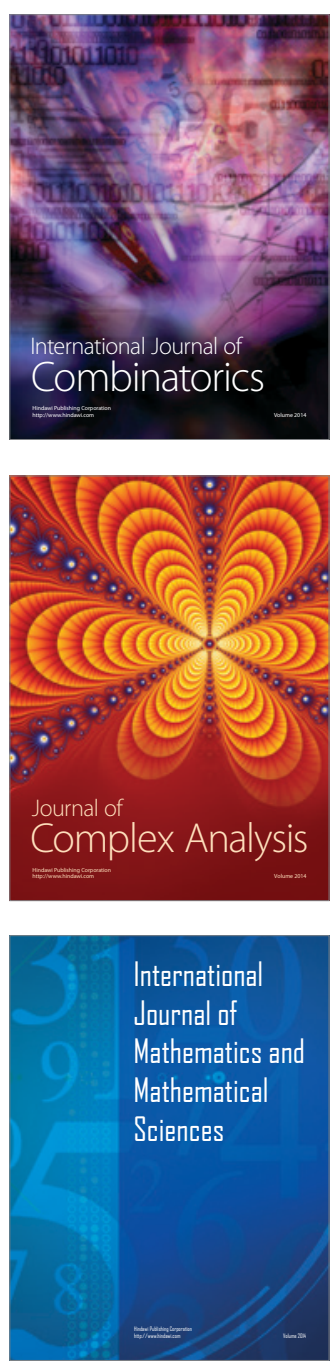
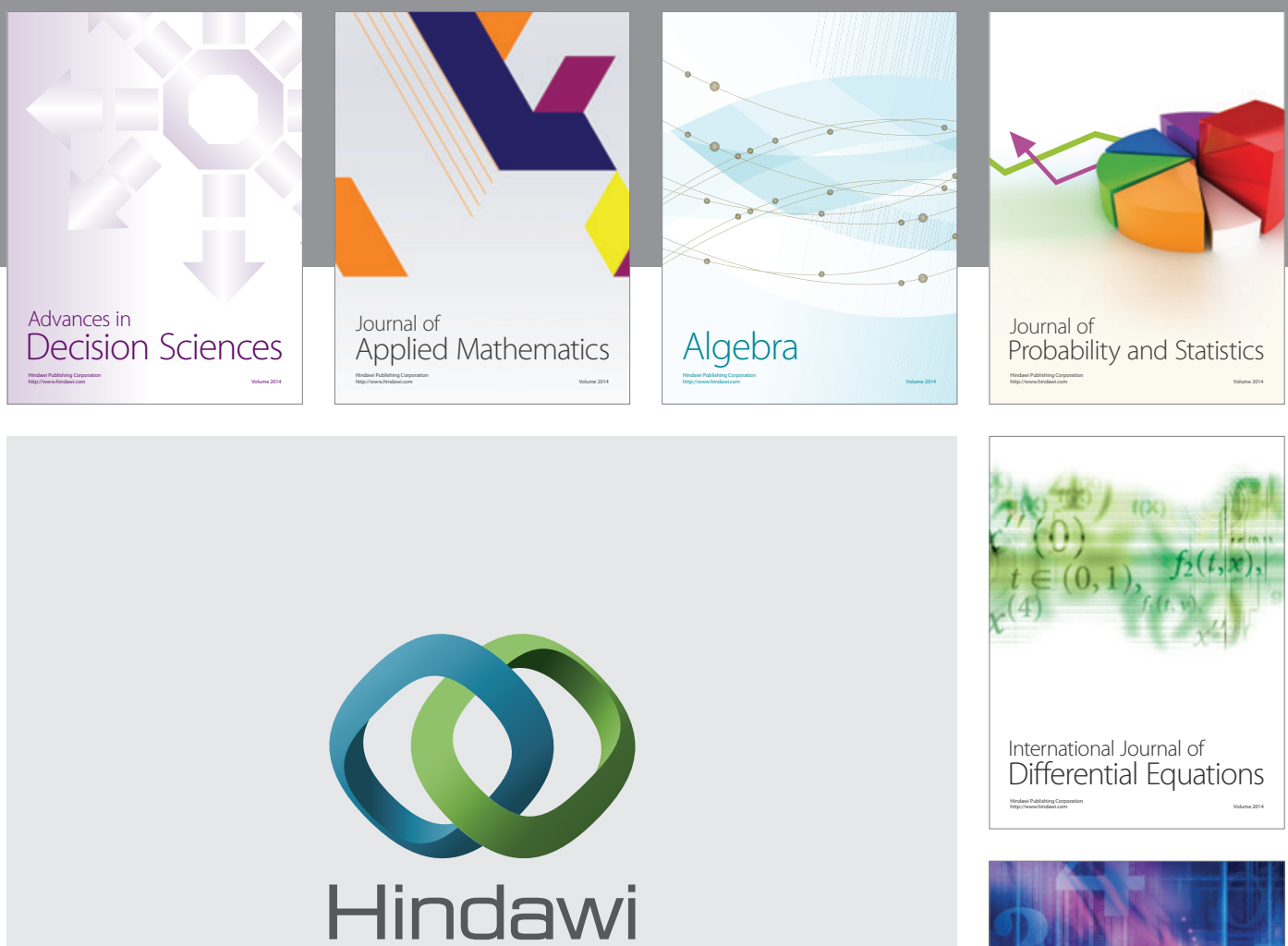

Submit your manuscripts at http://www.hindawi.com
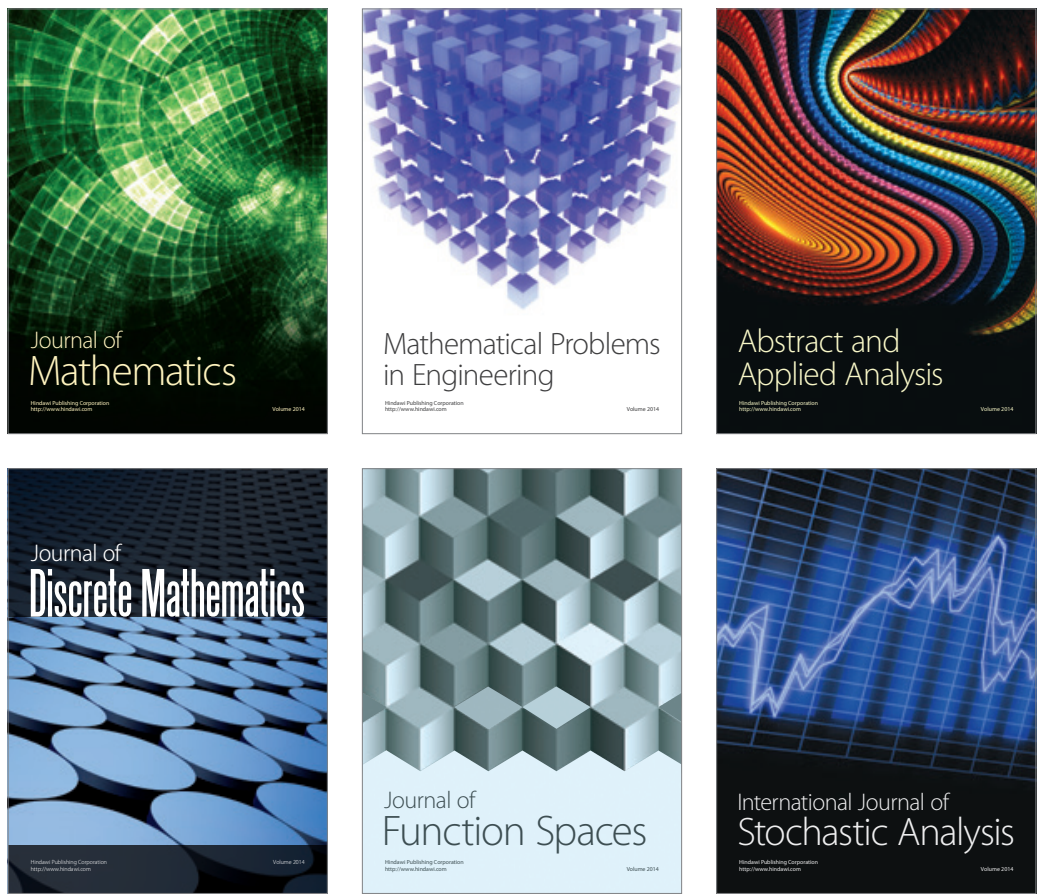

Journal of

Function Spaces

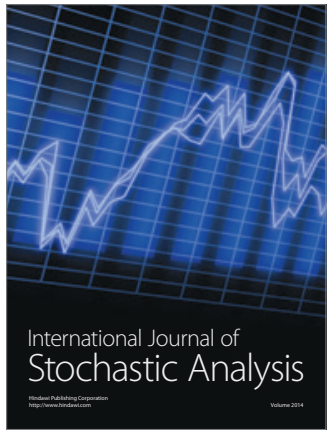

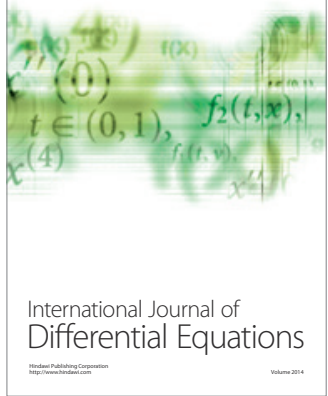
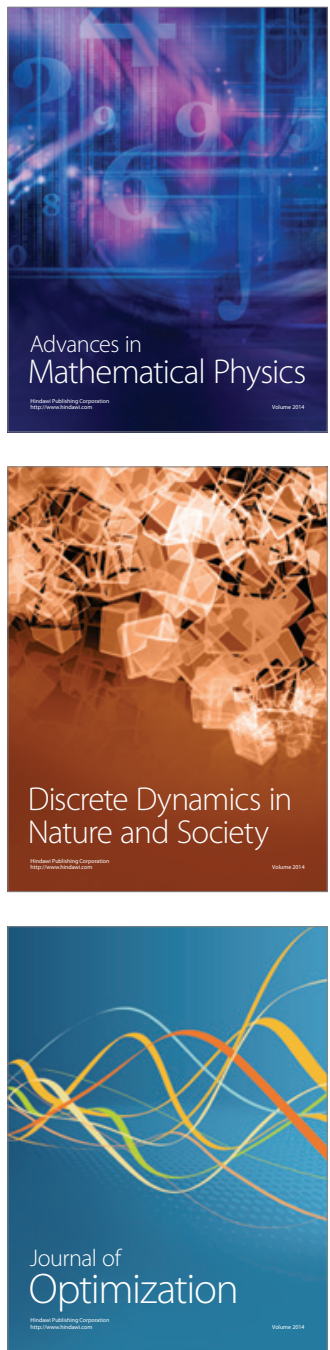